\title{
Label-free high-speed wide-field imaging of single microtubules using interference reflection microscopy
}

Mohammed Mahamdeh $^{1 *}$, Steve Simmert ${ }^{2 *}$, Anna Luchniak ${ }^{1}$, Erik Schäffer ${ }^{2}$ and Jonathon Howard ${ }^{1 \#}$

Addresses: ${ }^{1}$ Department of molecular biophysics and biochemistry, Yale university, New Haven, USA

${ }^{2}$ Center for plant molecular biology, University of Tübingen, Tübingen, Germany.

${ }^{*}$ Authors contributed equally to this work

\#Corresponding Author: jonathon.howard@yale.edu

266 Whitney Ave

PO Box 208114

New Haven CT 06520-8114

Phone: +1 (203) 432-5566

Fax: $+1(203) 432-8492$

Short title: Internal reflection imaging of single microtubules

Abbreviations:

DF: dark field, DIC: differential interference contrast, SBR: signal-to-background noise ratio, CSC: contrast sensitivity coefficient, FPS: frames per second, IRM: interference reflection microscopy, iScat: interferometric scattering, NA: numerical aperture, TIRF: total internal reflection, SLIM: spatial light interference microscopy, DU: digital unit. 


\section{Summary}

When studying microtubules in vitro, label free imaging of single microtubules is necessary when the quantity of purified tubulin is too low for efficient fluorescent labeling or there is concern that labelling will disrupt its function. Commonly used techniques for observing unlabeled microtubules, such as video enhanced differential interference contrast, dark-field and more recently laser-based interferometric scattering microscopy, suffer from a number of drawbacks. The contrast of differential interference contrast images depends on the orientation of the microtubules, dark-field is highly sensitive to impurities and optical misalignments, and interferometric scattering has a limited field of view. In addition, all of these techniques require costly optical components such as Nomarski prisms, dark-field condensers, lasers and laser scanners. Here we show that single microtubules can be imaged at high speed and with high contrast using interference reflection microscopy without the aforementioned drawbacks. Interference reflection microscopy is simple to implement, requiring only the incorporation of a 50/50 mirror instead of a dichroic in a fluorescence microscope, and with appropriate microscope settings has similar signal-to-noise ratio to differential interference contrast and fluorescence. We demonstrated the utility of interference reflection microscopy by high speed imaging and tracking of dynamic microtubules at 100 frames per second. In conclusion, the image quality of interference reflection microscopy is similar to or exceeds that of all other techniques and, with minimal microscope modification, can be used to study the dynamics of unlabeled microtubules. 


\section{INTRODUCTION}

In vitro imaging of single microtubules has proved to be an important tool to study microtubule dynamics (Hotani and Horio, 1988; Walker et al., 1988), mechanics (Dogterom and Yurke, 1997; Gittes et al., 1993), and how they are regulated (Gell et al., 2010) . Typically, fluorescence microscopy, in particular total internal reflection microscopy (TIRF), is used for imaging microtubule dynamics and gliding assays due to its high signal-to-background noise ratio (SBR, a measure of the optical contrast of the microtubule) and the wide range of available fluorescent labels (Gell et al. 2010). Efficient fluorescent labeling of tubulin requires a high tubulin concentration but often the yield of purified tubulin is too low (Widlund et al., 2012). In other cases, fluorescence-induced photo-damage is a concern (Vemu et al., 2016) especially if oxygen scavengers can react with the molecules of interest. In such cases, label free imaging is required.

There are many label-free imaging techniques available for imaging microtubules. Excellent techniques like video enhanced differential interference microscopy (DIC) and dark-field microscopy (DF) have been regularly used for almost 30 years (Bormuth et al., 2007; Gittes et al., 1993; Hotani and Horio, 1988; Walker et al., 1988). With DIC it is possible to image a large field of view at high frame rates. The drawback of using DIC is that the contrast of microtubules depends on their orientation with respect to the axis of the Nomarski prims. Although this can be solved (Shribak et al., 2008), the solution adds extra layers of complexity to the set up. Dark-field can visualize microtubule at high SBR assuming very clean solutions and surfaces. Otherwise, stray light caused by 
misalignment or light scattered by impurities will overwhelm the microtubule signal. Another technical inconvenience is that both techniques require the use of high numerical-aperture (NA) condensers that limit access to the sample during experiments. Other than DIC and dark-field, a number of techniques have emerged over the years. For example, Amos and Amos (Amos and Amos, 1991) imaged single microtubules by confocal reflection microscopy, requiring minimal modification to a confocal setup. Medina et al (Medina, 2010) visualized microtubules by defocusing the bright-field microscope, though the contrast was low even after image processing. Recently, Andrecka et al., used interferometric scattering (iSCAT) microscopy to image microtubule dynamics at high frame rates (Andrecka et al., 2016). iSCAT is capable of label-free imaging of single proteins but suffers from a limited field of view (practically on the order of $20 \times 20 \mu \mathrm{m}^{2}$ ) and requires a complex setup that involve lasers and laser scanners (Ortega Arroyo et al., 2016). Rotating-coherent-scattering microscopy (ROCS) which is also based on laser scattering was used to image microtubules far from the surface (Koch and Rohrbach, 2018). Just like iSCAT, ROSC requires using laser and scanners which complicates its implementation. Another recent technique uses spatial light interference microscopy (SLIM); while capable of imaging a large field of view $\left(200 \times 200 \mu \mathrm{m}^{2}\right)$ the frame rate is low (6.25 fps) (Kandel et al., 2017).

All the aforementioned techniques are capable of imaging single microtubules, but each fails to meet one or more of the following four criteria: (i) large field of view for higher throughput and to take advantage of the large chips of the new scientific CMOS cameras, (ii) high frame rate to improve temporal resolution, (iii) good signal-to- 
background noise ratio to achieve high spatial precision, and (iv) ease of implementation with low cost. In this work, we demonstrate that interference reflection microscopy (IRM) can image single label-free microtubules and satisfies all criteria. In IRM the image is formed by the interference between the light reflected from the glassmedium interface and that reflected from the medium-sample interface (Limozin and Sengupta, 2009; Weber, 2003). Since its first use in biology by Curtis in 1964 (Curtis, 1964), IRM, and the more advanced implementation known as reflective interference contrast microscopy, have been used intensively to probe cell adhesion to surfaces and other applications (Barr and Bunnell, 2001; Verschueren, 1985). Here we show that it is possible to image single microtubules using IRM in its simplest configuration.

\section{METHODS}

IRM was implemented by adding a 50/50 mirror (Chroma, Bellows Falls, VT USA) to a Nikon inverted fluorescence microscope (Ti Eclipse, Nikon, Melville, NY USA) in the position where usually a dichroic mirror is placed. No additional excitation or emission filters are needed. The sample was illuminated by a Sola light engine (Lumencore, Beaverton, OR USA) attached to the microscope's epi port. The NA of the illumination was set by the aperture iris. The field diaphragm was adjusted to optimize the contrast as presented in the Results section. The illumination light was partially reflected to the objective (100x/1.49 Apochromat, Nikon, Melville, NY USA) and the light reflected from the sample was collected by the same objective and projected -after passing through the $50 / 50$ mirror- onto a 16 bit sCMOS camera (Zyla 4.2, $6.5 \mu \mathrm{m}$ pixel size $(65 \mathrm{~nm}$ at image plane), 2048×2048 pixel $^{2}$ chip size, 72\% quantum efficiency, Andor, Belfast, 
Scotland). The illumination was adjusted to nearly saturate the camera's dynamic range. Experiments were performed in a flow channel formed by two parafilm strips sandwiched between $18 \times 18 \mathrm{~mm}^{2}$ and $22 \times 22 \mathrm{~mm}^{2}$ coverslips (\#1.5H, Marienfeld, Germany) or between a 22x22 $\mathrm{mm}^{2}$ coverslip and a one inch slide (Thermo Scientific, Waltham, MA USA) (Gell et al., 2010). The coverslips were cleaned using piranha solution and rendered hydrophobic by silanization (Gell et al., 2010). In non-dynamic assays GMPCPP stabilized, TAMRA labeled (tetramethylrhodamine Ex: $550 \mathrm{~nm}$, Em: $580 \mathrm{~nm}$ ) bovine microtubules were imaged at room temperature. The microtubules were either passively attached to the hydrophobic surface or fixed by an anti-TAMRA antibody (Life Technologies, Waltham, MA USA). For dynamic assays, the same configuration was used and microtubule growth was initiated by flowing in polymerization solution (80 mM PIPES/KOH, pH 6.9, 1 mM ethylene glycol tetra-acetic acid (EGTA), $1 \mathrm{mM} \mathrm{MgCl}, 2 \mathrm{mM} \mathrm{GTP}$ and $7.5 \mu \mathrm{M}$ unlabeled bovine tubulin) at $34^{\circ} \mathrm{C}$. Dynamic assays were imaged at slow (0.2 fps) and fast (100 fps) frame rates. In some dynamic assays the minus and plus end of the microtubules seeds were labeled using Alexa488 labeled (Ex: 490 nm, Em: 525 nm) tubulin. In all IRM imaging a long pass filter (LP 594 nm, Chroma, Bellows Falls, VT USA) was inserted into the illumination path to avoid exciting the fluorophores. Such a filter is unnecessary if unlabeled tubulin is used. No anti-fade reagents (Gell et al., 2010) were used.

To enhance contrast and eliminate illumination irregularities and static noise, a background image was subtracted from the acquired images. The background image was generated by averaging 32 or 100 images acquired before flowing in the 
microtubules or it was the median of a 100 images acquired while moving the sample at a high speed after imaging. Further enhancement of images included averaging, Fourier filtering or both (Bormuth et al., 2007). All image processing was performed using Fiji (Schindelin et al., 2012). Tracking of microtubules was done using the tracking software FIESTA (Ruhnow et al., 2011).

For DIC imaging of microtubules, a high NA oil immersion condenser equipped with a high resolution prism (NA = 1.4, Nikon, Melville, NY USA) was used for illuminating the sample. To enhance the contrast of the images, a bias retardation of one tenth of the wavelength was applied. Background images were generated by averaging 100 images taken 1-2 $\mu \mathrm{m}$ from the surface. As for IRM images, the images could be further enhanced by averaging and/or filtering.

Fluorescence images were obtained by TIRF microscopy. The microtubules were excited by a $561 \mathrm{~nm}$ laser at a power of $\sim 1 \mathrm{~mW}$ (at the sample plane) with an exposure time of $100 \mathrm{~ms}$.

As a metric to assess IRM and compare it to different techniques we measured the signal-to-background noise ratio of a microtubule as defined by the average intensity of the microtubule signal (intensity of the microtubule minus the intensity of the background) divided by the standard deviation of the background. The background was defined in a region close to the microtubule, as described below.

For IRM and TIRF images, the signal was obtained by first isolating the microtubule in a rectangle region of interest $(\mathrm{ROI})$ that was slightly longer than the microtubule along its 
axis and 60 pixels in the perpendicular direction. Next, the cross section profile of the microtubule was measured by taking a line scan perpendicular to the microtubule axis. The line width was set to equal the microtubule length. This way, every point on the cross section profile was an average of all pixels along the microtubule axis. Then, the signal was measured as the difference between the profile peak and the background. The background was measured by thresholding the microtubule image to separate the microtubule from the background and then averaging all pixels below the cut off intensity. In the case of DIC images, the signal-to-background noise ratio was determined as the peak to peak difference of the averaged signal divided by the standard deviation of the background noise (Bormuth et al., 2007).

We also measured the contrast sensitivity coefficient (CSC) define as the microtubule signal divided by the standard deviation of the microtubule signal. Here, the signal is the mean of all the pixels' values above the threshold cut off after background subtraction. The noise is the standard deviation of the signal. CSC measurement is applicable to IRM and TIRF microtubule images but not DIC because the standard deviation along the microtubule will be higher than the signal. This is because the signal will nearly vanish since the intensities above and below the background tend to cancel each other.

\section{RESULTS AND DISCUSSION}

Microtubules are readily visible in IRM without background subtraction. The microtubules appeared dark against a brighter background (Fig, 2a). Reducing the field of view size by the field diaphragm reduced the amount of stray light reaching the 
camera and improved the contrast of the microtubule (Fig. 1a). We found that opening the field diaphragm up to $70 \%$ of the field of view was a good balance between improving contrast and maintaining a large imaging area. In addition to the field diaphragm, the contrast depended on the illumination NA (Weber, 2003). Qualitatively, without background subtraction, the microtubules visibility was highest in the NA range 0.7-1.1 (Fig. 1b). This agreed with SBR measurements of background-subtracted images as a function of illumination NA (Fig. 1c). The SBR of a single frame of background-subtracted images was $6.8 \pm 0.8$ (mean $\pm S D, N=41)$. To further improve the SBR, the images were either averaged or filtered or both. Upon averaging, the SBR initially increased with a power law of 0.25 , less than the 0.5 dependence expected for a photon-shot-noise limited image. This deviation can be explained by the presence of other sources of noise such as dirt and drift. As the number of averaged frames increased, the SBR saturated as the noise in the image was no further reduced by averaging (supplementary Fig, 2a). By careful examination of the noise, we found that as the number of averaged frames approached the number of averaged background images the gained noise reduction saturated (supplementary Fig, 2b). The average of 10 images, increased the SBR to $11.6 \pm 1.6$. A comparable increase to $11.2 \pm 1.2$ was obtained by using a low-pass Fourier filter. Filtering the averaged images results in a final SBR of $17.4 \pm 2.4$ (Fig, 2).

To compare the performance of IRM to other techniques, the same field of view of microtubules was imaged by IRM, TIRF and DIC and the SBR for each image was measured (Fig, 3a). For TIRF of highly labelled microtubules (30\%, to reduce speckles 
(Waterman-Storer et al., 1998)), the SBR was $40 \pm 10$ (mean $\pm S D, N=41)$ prior to photobleaching. In terms of CSC, both IRM and TIRF images were similar, CSC TIRF= $2.64 \pm 0.13$ (mean $\pm S D$ ) compared to $\mathrm{CSC}_{\mathrm{IRM}}=2.7 \pm 0.18$. DIC images had a lower SBR $(10 \pm 2)$ than IRM. Aside from the higher SBR, the advantage of using IRM over DIC is that the contrast is independent of microtubule orientation as can be clearly seen in Fig, 3a. Such dependence has an impact when tracking microtubules imaged in DIC (Danuser et al., 2000; Janson and Dogterom, 2004).

To demonstrate the capability of IRM, dynamic microtubules were imaged. Averaged background-subtracted images were taken at a frame rate of $0.2 \mathrm{fps}$. Growth and shrinkage phases where easily observed in the videos and kymographs (Fig, 3b, Supplementary video 1). While such low frame rate is suitable for measuring growth rate and catastrophe frequency, it is more challenging to measure the shrinkage rates which are an order of magnitude higher than growth rates. For this reason, shrinking microtubules were imaged at $100 \mathrm{fps}$ which provided a detailed image of the shrinking end (Fig, 3b). At both frame rates, it was possible to track the microtubule ends using the tracking software FIESTA with a length precision of $\approx 20 \mathrm{~nm}$ (Supplementary video 1). In addition, IRM can be easily combined with fluorescence microscopy, thus freeing one of the fluorescence channels to image, for example, molecular motors, MAPs or specially modified tubulins (Fig, 3c).

In TIRF and DIC, thermal fluctuations of long microtubules away from the surface lead to loss of contrast. In IRM, such fluctuations lead to a reverse in microtubule contrast, 
which can be used to measure the height of the microtubule, similar to fluorescence interference contrast microscopy (Kerssemakers et al., 2006; Lambacher and Fromherz, 1996) (supplementary video 2). Recently, the ability to measure distances from the surface using IRM was used as a mean to calibrate the TIRF evanescent field in a combined IRM-TIRF-optical tweezers setup using a light emitting diode for illumination (Simmert et al., 2018). It is also worth mentioning that IRM is flexible in terms of what objective to use: it was possible to image microtubules using high end and low end objectives as well as phase and DIC objectives. The presence of a Nomarski prism or the phase ring did not seem to influence the images.

The main drawback of IRM is its sensitivity to drift which is noticeable when imaging for extended periods of time (Supplementary Fig, 3). This requires the setup to be thermally stabilized (Mahamdeh and Schäffer, 2009) or the image to be drift corrected (Carter et al., 2007; Kim and Saleh, 2008; Ortega Arroyo et al., 2016).

In conclusion, IRM proved to be a powerful tool for imaging unlabeled microtubules in surface assays. It only requires a minor, cost effective modification to any epimicroscope, requires a onetime alignment, it is capable of high-speed wide-field (100 fps at full chip) imaging of microtubules at a high spatial precision for long periods of time and can be combined easily with other techniques. 


\section{References}

Amos, L.A., and Amos, W.B. (1991). The bending of sliding microtubules imaged by confocal light microscopy and negative stain electron microscopy. J Cell Sci 1991, 95-101.

Andrecka, J., Ortega Arroyo, J., Lewis, K., Cross, R.A., and Kukura, P. (2016). Label-free Imaging of Microtubules with Sub-nm Precision Using Interferometric Scattering Microscopy. Biophys. J. 110, 214217.

Barr, V.A., and Bunnell, S.C. (2001). Interference Reflection Microscopy. In Current Protocols in Cell Biology, (John Wiley \& Sons, Inc.), p.

Bormuth, V., Howard, J., and Schäffer, E. (2007). LED illumination for video-enhanced DIC imaging of single microtubules. J Microsc 226, 1-5.

Carter, A.R., King, G.M., Ulrich, T.A., Halsey, W., Alchenberger, D., and Perkins, T.T. (2007). Stabilization of an optical microscope to $0.1 \mathrm{~nm}$ in three dimensions. Appl Opt 46, 421-427.

Curtis, A.S.G. (1964). The Mechanism of Adhesion of Cells to Glass. J. Cell Biol. 20, 199-215.

Danuser, G., Tran, P.T., and Salmon, E.D. (2000). Tracking differential interference contrast diffraction line images with nanometre sensitivity. J. Microsc. 198, 34-53.

Dogterom, M., and Yurke, B. (1997). Measurement of the Force-Velocity Relation for Growing Microtubules. Science 278, 856-860.

Gell, C., Bormuth, V., Brouhard, G.J., Cohen, D.N., Diez, S., Friel, C.T., Helenius, J., Nitzsche, B., Petzold, H., Ribbe, J., et al. (2010). Chapter 13 - Microtubule Dynamics Reconstituted In Vitro and Imaged by Single-Molecule Fluorescence Microscopy. In Methods in Cell Biology, L.W. and J.J. Correia, ed.

(Academic Press), pp. 221-245.

Gittes, F., Mickey, B., Nettleton, J., and Howard, J. (1993). Flexural rigidity of microtubules and actin filaments measured from thermal fluctuations in shape. J. Cell Biol. 120, 923-934.

Hotani, H., and Horio, T. (1988). Dynamics of microtubules visualized by darkfield microscopy:

Treadmilling and dynamic instability. Cell Motil. Cytoskeleton 10, 229-236.

Janson, M.E., and Dogterom, M. (2004). A Bending Mode Analysis for Growing Microtubules: Evidence for a Velocity-Dependent Rigidity. Biophys. J. 87, 2723-2736.

Kandel, M.E., Teng, K.W., Selvin, P.R., and Popescu, G. (2017). Label-Free Imaging of Single Microtubule Dynamics Using Spatial Light Interference Microscopy. ACS Nano 11, 647-655.

Kerssemakers, J., Howard, J., Hess, H., and Diez, S. (2006). The distance that kinesin-1 holds its cargo from the microtubule surface measured by fluorescence interference contrast microscopy. Proc. Natl. Acad. Sci. 103, 15812-15817.

Kim, K., and Saleh, O.A. (2008). Stabilizing method for reflection interference contrast microscopy. Appl. Opt. 47, 2070-2075. 
Koch, M.D., and Rohrbach, A. (2018). Label-free Imaging and Bending Analysis of Microtubules by ROCS Microscopy and Optical Trapping. Biophys. J. 114, 168-177.

Lambacher, A., and Fromherz, P. (1996). Fluorescence interference-contrast microscopy on oxidized silicon using a monomolecular dye layer. Appl. Phys. A 63, 207-216.

Limozin, L., and Sengupta, K. (2009). Quantitative Reflection Interference Contrast Microscopy (RICM) in Soft Matter and Cell Adhesion. ChemPhysChem 10, 2752-2768.

Mahamdeh, M., and Schäffer, E. (2009). Optical tweezers with millikelvin precision of temperaturecontrolled objectives and base-pair resolution. Opt. Express 17, 17190-17199.

Ortega Arroyo, J., Cole, D., and Kukura, P. (2016). Interferometric scattering microscopy and its combination with single-molecule fluorescence imaging. Nat. Protoc. 11, 617-633.

Ruhnow, F., Zwicker, D., and Diez, S. (2011). Tracking Single Particles and Elongated Filaments with Nanometer Precision. Biophys. J. 100, 2820-2828.

Schindelin, J., Arganda-Carreras, I., Frise, E., Kaynig, V., Longair, M., Pietzsch, T., Preibisch, S., Rueden, C., Saalfeld, S., Schmid, B., et al. (2012). Fiji: an open-source platform for biological-image analysis. Nat. Methods 9, 676-682.

Shribak, M., LaFountain, J., Biggs, D., and Inouè, S. (2008). Orientation-independent differential interference contrast microscopy and its combination with an orientation-independent polarization system. J. Biomed. Opt. 13, 014011-014011-10.

Simmert, S., Abdosamadi, M.K., and Schäffer, E. (2018). LED-based interference reflection and total internal reflection microscopy combined with optical tweezers for quantitative 3-dimensional imaging of single microtubules. BIORXIV/2018/277632.

Vemu, A., Atherton, J., Spector, J.O., Szyk, A., Moores, C.A., and Roll-Mecak, A. (2016). Structure and Dynamics of Single-isoform Recombinant Neuronal Human Tubulin. J. Biol. Chem. 291, 12907-12915.

Verschueren, H. (1985). Interference reflection microscopy in cell biology: methodology and applications. J. Cell Sci. 75, 279-301.

Walker, R.A., O’Brien, E.T., Pryer, N.K., Soboeiro, M.F., Voter, W.A., Erickson, H.P., and Salmon, E.D. (1988). Dynamic instability of individual microtubules analyzed by video light microscopy: rate constants and transition frequencies. J. Cell Biol. 107, 1437-1448.

Waterman-Storer, C.M., Desai, A., Chloe Bulinski, J., and Salmon, E.D. (1998). Fluorescent speckle microscopy, a method to visualize the dynamics of protein assemblies in living cells. Curr. Biol. 8, 1227S1.

Weber, I. (2003). [2] Reflection interference contrast microscopy. Methods Enzymol. 361, 34-47.

Widlund, P.O., Podolski, M., Reber, S., Alper, J., Storch, M., Hyman, A.A., Howard, J., and Drechsel, D.N. (2012). One-step purification of assembly-competent tubulin from diverse eukaryotic sources. Mol. Biol. Cell 23, 4393-4401. 
bioRxiv preprint doi: https://doi.org/10.1101/273086; this version posted March 6, 2018. The copyright holder for this preprint (which was not certified by peer review) is the author/funder, who has granted bioRxiv a license to display the preprint in perpetuity. It is made available under aCC-BY-NC-ND 4.0 International license. 
bioRxiv preprint doi: https://doi.org/10.1101/273086; this version posted March 6, 2018. The copyright holder for this preprint (which was not certified by peer review) is the author/funder, who has granted bioRxiv a license to display the preprint in perpetuity. It is made available under aCC-BY-NC-ND 4.0 International license.

A

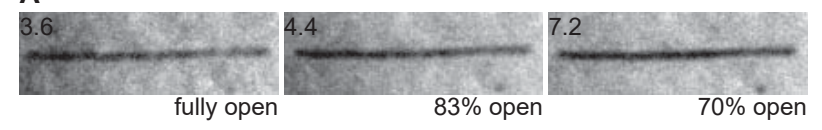

B

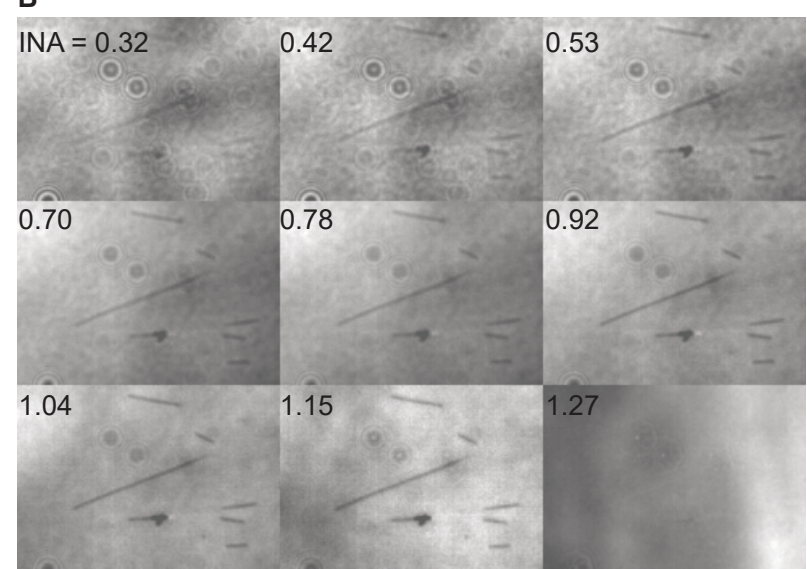

C

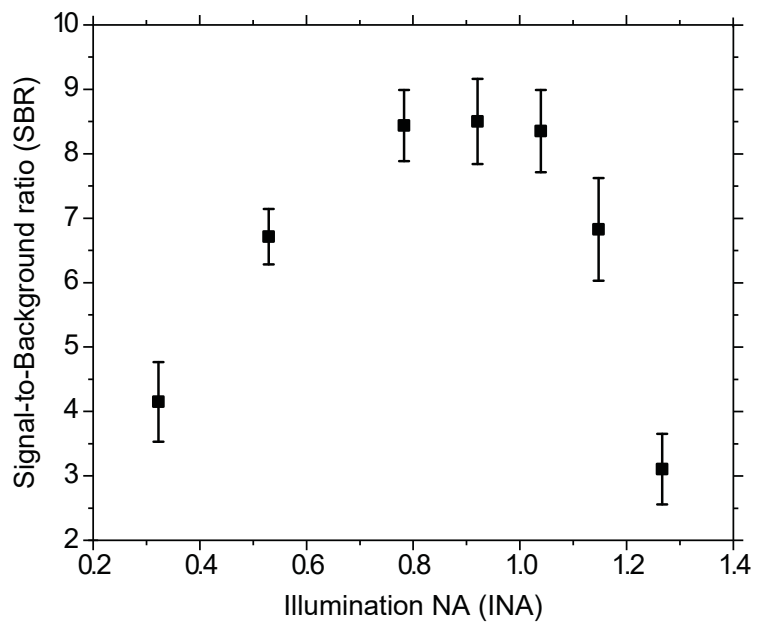


bioRxiv preprint doi: https://doi.org/10.1101/273086; this version posted March 6, 2018. The copyright holder for this preprint (which was not certified by peer review) is the author/funder, who has granted bioRxiv a license to display the preprint in perpetuity. It is made available under aCC-BY-NC-ND 4.0 International license.
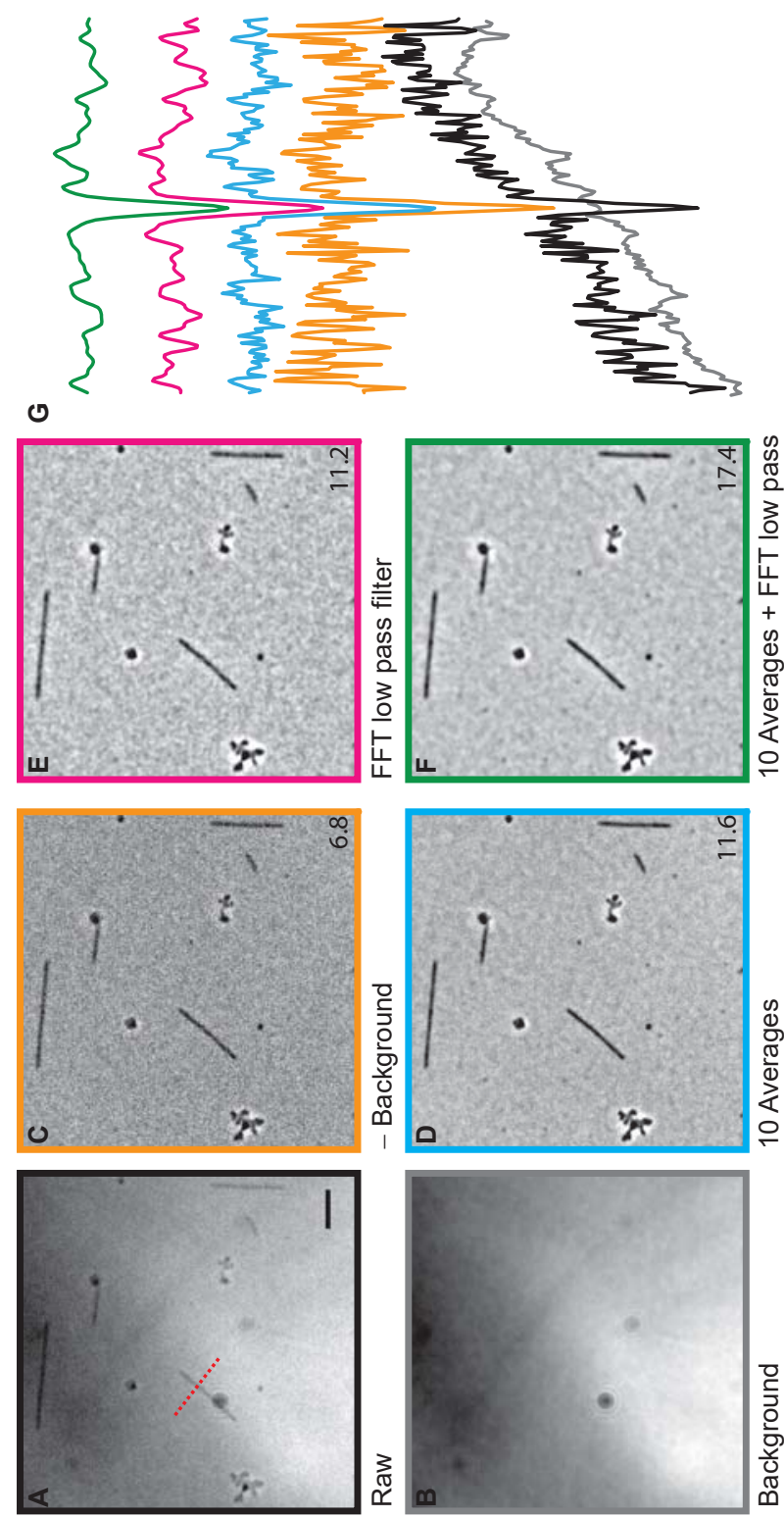
bioRxiv preprint doi: https://doi.org/10.1101/273086; this version posted March 6, 2018. The copyright holder for this preprint (which was not certified by peer review) is the author/funder, who has granted bioRxiv a license to display the preprint in perpetuity. It is made available under aCC-BY-NC-ND 4.0 International license.

\section{A}

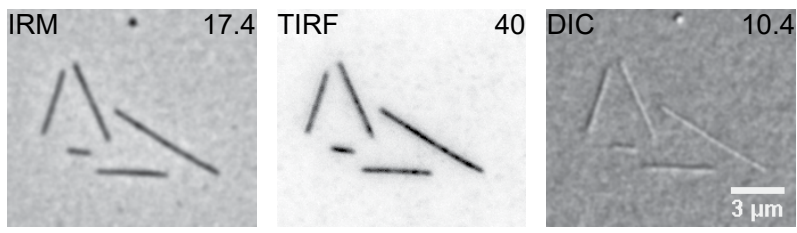

B

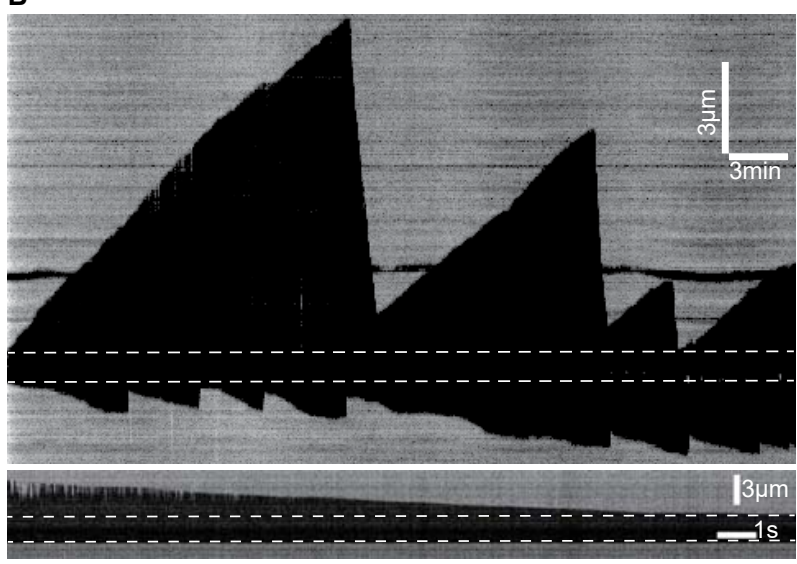

C

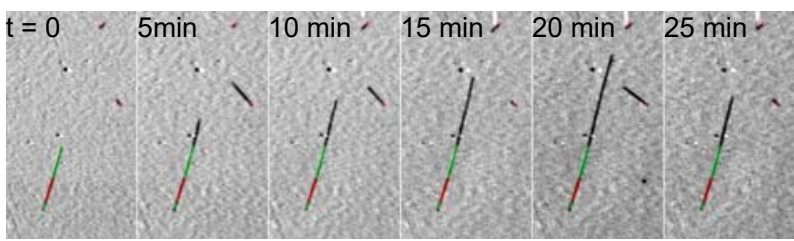

